\title{
Monotonicity of the eigenvalues of the two-particle Schrödinger operatoron a lattice
}

\author{
J. I. Abdullaev ${ }^{1,2}$, A. M. Khalkhuzhaev ${ }^{1,2}$, L. S. Usmonov ${ }^{2}$ \\ ${ }^{1}$ Institute of Mathematics of the Academy of Sciences of the Republic of Uzbekistan, \\ Mirzo Ulugbek 81, Tashkent 100170, Uzbekistan \\ ${ }^{2}$ Samarkand State University, University Boulevard 15, Samarkand 140104, Uzbekistan \\ jabdullaev@mail.ru, ahmad_x@mail.ru, u.lochinbek@bk.ru
}

PACS 02.30.Tb

DOI 10.17586/2220-8054-2021-12-6-657-663

\begin{abstract}
We consider the two-particle Schrödinger operator $H(\mathbf{k}),\left(\mathbf{k} \in \mathbf{T}^{3} \equiv(-\pi, \pi]^{3}\right.$ is the total quasimomentum of a system of two particles) corresponding to the Hamiltonian of the two-particle system on the three-dimensional lattice $\mathbf{Z}^{3}$. It is proved that the number $N(\mathbf{k}) \equiv N\left(k^{(1)}, k^{(2)}, k^{(3)}\right)$ of eigenvalues below the essential spectrum of the operator $H(\mathbf{k})$ is nondecreasing function in each $k^{(i)} \in[0, \pi], i=1,2,3$. Under some additional conditions potential $\hat{v}$, the monotonicity of each eigenvalue $z_{n}(\mathbf{k}) \equiv z_{n}\left(k^{(1)}, k^{(2)}, k^{(3)}\right)$ of the operator $H(\mathbf{k})$ in $k^{(i)} \in[0, \pi]$ with other coordinates $\mathbf{k}$ being fixed is proved.
\end{abstract}

Keywords: two-particle Schrödinger operator, Birman-Schwinger principle, total quasimomentum, monotonicity of the eigenvalues.

Received: 22 October 2021

Revised: 20 November 2021

\section{Introduction}

Coherent optical fields provide a strong tool for manipulating ultracold atoms and a unique setting for quantum simulations of interacting many-body systems because of high-degree of controllable parameters such as optical lattice geometry and dimensionality, particle masses, tunneling, two-body potentials, temperature etc. [1-4]. However, in such manipulations, due to diffraction, there is a fundamental limit for the length scale, given by the wavelength of light [5] and therefore, the corresponding models are naturally restricted to the short-range case. However, recent experimental and theoretical results show that integrating plasmonic systems with cold atoms, especially using optical potential fields formed from the near field scattering of light by an array of plasmonic nanoparticles allows one to considerably increase the energy scales in the realization of Hubbard models and engineer effective long-range interaction in many body dynamics [5-7].

Hamiltonians, corresponding to systems of particles on a lattice, were first considered in the 1990s by D. S. Mattis [8], A. I. Mogilner [9], and after that, research has rapidly developed. The kinematics of quantum quasiparticles on a lattice is rather peculiar, even in the two-particle case. For example, because the discrete analog of the Laplacian or of its generalization is not translation invariant, the Hamiltonian of the system cannot be divided into two parts with one part corresponding to the motion of the center and the other corresponding to the internal degrees of freedom. This is the so-called phenomenon of "excess mass" for lattice systems: the effective mass of the two-particle bound state exceeds the sum of the effective masses of the quasiparticles constituting the system (see, e.g., [8,9]). In contrast to the continuous case, where the center-of-mass motion can be separated, the two-particle problem on a lattice reduces to studying the one-particle problem using the Gelfand transformation. Namely, the Hilbert space $\ell_{2}\left(\left(\mathbf{Z}^{3}\right)^{2}\right)$ can be decomposed into the direct (continuous) von Neumann integral associated with the representation of the Abelian (discrete) group $\mathbf{Z}^{3}$ formed by commutative operators on the lattice. Then the two-particle Hamiltonian can also be decomposed into the direct (continuous) von Neumann integral. In contrast to the continuous case, the corresponding fiber operators $H(\mathbf{k}), \mathbf{k} \in \mathbf{T}^{3}$, associated with the decomposition of the direct integral depend parametrically on the quasimomentum $\mathbf{k}$, which ranges the first Brillouin zone $\mathbf{R}^{3} \backslash(2 \pi \mathbf{Z})^{3}$. Because the spherical symmetry of the problem is lost, the spectra of the family $H(\mathbf{k}), \mathbf{k} \in \mathbf{T}^{3}$, become rather sensitive to variations in the quasimomentum $\mathbf{k}$.

Spectral properties of the two-particle discrete Schrödinger operator $H(\mathbf{k})=H_{0}(\mathbf{k})-V, \mathbf{k} \in \mathbf{T}^{3}$ are studied in the more works (see.i.e. [10-13]). In work [13] a two-particle discrete Schrödinger operator $H(\mathbf{k}), \mathbf{k} \in \mathbf{T}^{3}$ with zero range potential $\hat{v}\left(\mathbf{n}_{1}-\mathbf{n}_{2}\right)=\mu \delta_{\mathbf{n}_{1} \mathbf{n}_{\mathbf{2}}}$ was considered and the existence of a unique eigenvalue $z(\mathbf{k})$ of the operator $H(\mathbf{k})$ was established. In [13] it is proved that the eigenvalue $z(\mathbf{k})=z\left(k^{(1)}, k^{(2)}, k^{(3)}\right), \mathbf{k} \in \mathbf{T}^{3}$ is symmetric and even in each variable $k^{(i)} \in[-\pi, \pi], i=1,2,3$ and strictly increases in each $k^{(i)} \in[0, \pi], i=1,2,3$. In particular, it was shown that the two-particle operator $H(\mathbf{k}), \mathbf{k} \neq \mathbf{0}$ has a positive eigenvalue below the essential spectrum, provided that $H(\mathbf{0})$ has a virtual level at zero. 
The following effect was discovered in [14] for a wide class of two-particle Schrödinger operators $H(\mathbf{k})$, associated with the Hamiltonian of the system of two arbitrary particles. If the discrete Schrödinger operator $H(\mathbf{0})$, $\mathbf{0}=(0,0,0) \in \mathbf{T}^{3}$, has a virtual level or an eigenvalue on the lower threshold of the essential spectrum, then the operator $H(\mathbf{k})$, has an eigenvalue below the threshold of the essential spectrum for all nonzero values of the quasimomentum $\mathbf{k} \in \mathbf{T}^{3}$. Similar results was discussed in [15] for $d$-dimensional lattice case. In [16] was studied the discrete spectrum of the two-particle Schrödinger operator $H_{\mu, \lambda}(\mathbf{k}), \mathbf{k} \in \mathbf{T}^{2}$, associated to the Bose-Hubbard Hamiltonian $\hat{H}_{\mu, \lambda}$ of a system of two identical bosons interacting on site and nearest-neighbor sites in the two dimensional lattice $\mathbf{Z}^{2}$, with interaction magnitudes $\mu \in \mathbb{R}$ and $\lambda \in \mathbb{R}$, respectively, and completely described the spectrum of $H_{\mu, \lambda}(\mathbf{0})$ and established the optimal lower bound for the number of eigenvalues of $H_{\mu, \lambda}(\mathbf{k})$ outside its essential spectrum for all values of $\mathbf{k} \in \mathbf{T}^{2}$. Namely, the $(\mu, \lambda)$-plane was partitioned that in each connected component of the partition, the number of bound states of below or above its essential spectrum cannot be less than the corresponding number of bound states of $H_{\mu, \lambda}(\mathbf{0})$ below or above its essential spectrum. In [17] a two-particle Schrödinger operator $H$ on the $d$ - dimensional diamond lattice was considered and a sufficiency condition of finiteness for discrete spectrum eigenvalues of $H$ was found.

In this note, we consider the two-particle operator $H(\mathbf{k})=H_{0}(\mathbf{k})-V, \mathbf{k} \in \mathbf{T}^{3}$ with general potential $v$. For the potential $\hat{v}(\mathbf{x})=(\mathcal{F} v)(\mathbf{x})$ we assume:

$$
\hat{v}(\mathbf{x}) \geq 0, \forall \mathbf{x} \in \mathbf{Z}^{3}, \quad \hat{v} \in \ell_{1}\left(\mathbf{Z}^{3}\right) .
$$

Non-negativity $\hat{v}(\mathbf{x}) \geq 0$ will ensure the positivity interaction operator $V$. We denote by $V^{1 / 2}$ its positive square root. When proving monotonicity the eigenvalue $z_{n}(\mathbf{k})$ of the operator $H(\mathbf{k})$ with respect to $k^{(i)} \in[0, \pi]$, we will use the monotonicity property of the operator-valued function:

$$
G(\mathbf{k}, z)=V^{\frac{1}{2}}\left(H_{0}(\mathbf{k})-z I\right)^{-1} V^{\frac{1}{2}}
$$

by $z \in\left(-\infty, \mathcal{E}_{\min }(\mathbf{k})\right)$ and $k^{(i)} \in[0, \pi]$, where the number $\mathcal{E}_{\min }(\mathbf{k})$ is the left edge of the essential spectrum of the operator $H(\mathbf{k})$. For any $\mathbf{k} \in(-\pi, \pi)^{3}$ the operator $G(\mathbf{k}, z)$ converges uniformly to the limit operator $G\left(\mathbf{k}, \mathcal{E}_{\min }(\mathbf{k})\right)$ as $z \rightarrow \mathcal{E}_{\min }(\mathbf{k})$. Under the condition (1.1), it is proved that $G\left(\mathbf{k}, \mathcal{E}_{\min }(\mathbf{k})\right.$ ) belongs to the class $\Sigma_{1}$ (see. proof of the Lemma 3.1.) Since $G\left(\mathbf{k}, \mathcal{E}_{\min }(\mathbf{k})\right)$ is monotonic in each $k^{(i)} \in[0, \pi], i=1,2,3$ it follows that the number $N(\mathbf{k})$ of eigenvalues lying below the essential spectrum of the operator $H(\mathbf{k})$ is nondecreasing function with respect in each $k^{(i)} \in[0, \pi], i=1,2,3$ (Theorem 2.1).

We will prove the monotonicity $G(\mathbf{k}, z)$ by $z \in\left(-\infty, \mathcal{E}_{\min }(\mathbf{k})\right)$, that is $G\left(\mathbf{k}, z_{1}\right) \leq G\left(\mathbf{k}, z_{2}\right)$ at $z_{1}<z_{2}$. This implies that each eigenvalue $\lambda_{n}(\mathbf{k}, z)$ of the operator $G(\mathbf{k}, z)$ is increasing function with respect to $z \in\left(-\infty, \mathcal{E}_{\min }(\mathbf{k})\right)$ (Lemma 3.4). Given $\hat{v}\left(2 s, n^{(2)}, n^{(3)}\right) \equiv 0, \forall s \in \mathbf{Z}$ or $\hat{v}\left(2 s+1, n^{(2)}, n^{(3)}\right) \equiv 0, \forall s \in \mathbf{Z}$ the operator-valued function $G(\mathbf{k}, z)$ decreases by $k^{(1)} \in[0, \pi]$ (Lemma 3.5). It follows that each eigenvalue $z_{n}(\mathbf{k})$ of the two-particle operator $H(\mathbf{k})$ increases in $k^{(1)} \in[0, \pi]$ (Theorem 2.3).

\section{Representation of Hamiltonian associated to a system of two particle on a lattice. Statement of the main result}

Energy operator $\hat{H}$ of a system of two quantum particles on a three-dimensional lattice $\mathbf{Z}^{3}$ acts in the Hilbert space $\ell_{2}\left(\left(\mathbf{Z}^{3}\right)^{2}\right)$ by:

$$
\hat{H}=\hat{H}_{0}-\hat{V}
$$

where the free energy operator $\hat{H}_{0}$ acts in $\ell_{2}\left(\left(\mathbf{Z}^{3}\right)^{2}\right)$ as:

$$
\hat{H}_{0}=-\frac{1}{2 m_{1}} \Delta_{x_{1}}-\frac{1}{2 m_{2}} \Delta_{x_{2}}
$$

Here, $m_{1}, m_{2}>0$ are denoted the masses of particles, which in the future are considered equal to one, $\Delta_{x_{1}}=\Delta \otimes I$ and $\Delta_{x_{2}}=I \otimes \Delta$, lattice Laplacian $\Delta$ is a difference operator describing the transfer of a particle from a site to neighboring site:

$$
(\Delta \hat{\psi})(\mathbf{x})=\sum_{i=1}^{3}\left[\hat{\psi}\left(\mathbf{x}+\mathbf{e}_{i}\right)+\hat{\psi}\left(\mathbf{x}-\mathbf{e}_{i}\right)-2 \hat{\psi}(\mathbf{x})\right], \quad \hat{\psi} \in \ell_{2}\left(\mathbf{Z}^{3}\right)
$$

where $\mathbf{e}_{1}=(1,0,0), \mathbf{e}_{2}=(0,1,0), \mathbf{e}_{3}=(0,0,1)$ are the unit vectors in $\mathbf{Z}^{3}$.

The interaction of two particles is described by the operator $\hat{V}$ :

$$
(\hat{V} \hat{\psi})\left(\mathbf{x}_{\mathbf{1}}, \mathbf{x}_{\mathbf{2}}\right)=\hat{v}\left(\mathbf{x}_{\mathbf{1}}-\mathbf{x}_{\mathbf{2}}\right) \hat{\psi}\left(\mathbf{x}_{\mathbf{1}}, \mathbf{x}_{\mathbf{2}}\right), \quad \hat{\psi} \in \ell_{2}\left(\left(\mathbf{Z}^{3}\right)^{2}\right) .
$$


Under the conditions (1.1), the energy operator $\hat{H}$ is the bounded self-adjoint operator in the space $\ell_{2}\left(\left(\mathbf{Z}^{3}\right)^{2}\right)$. Transition to momentum representation is performed by using the Fourier transform $\mathcal{F}: L_{2}\left(\left(\mathbf{T}^{3}\right)^{2}\right) \rightarrow \ell_{2}\left(\left(\mathbf{Z}^{3}\right)^{2}\right)$. Operator energy $H=\mathcal{F}^{-1} \hat{H} \mathcal{F}$ in the momentum representation commutes with the group of unitary operators $U_{\mathbf{s}}, \mathbf{s} \in \mathbf{Z}^{3}$ :

$$
\left(U_{\mathbf{s}} f\right)\left(\mathbf{k}_{\mathbf{1}}, \mathbf{k}_{\mathbf{2}}\right)=\exp \left(-i\left(\mathbf{s}, \mathbf{k}_{\mathbf{1}}+\mathbf{k}_{\mathbf{2}}\right)\right) f\left(\mathbf{k}_{\mathbf{1}}, \mathbf{k}_{\mathbf{2}}\right), \quad f \in L_{2}\left(\left(\mathbf{T}^{3}\right)^{2}\right) .
$$

From the last fact we obtain [18] that there are decompositions of the space $L_{2}\left(\left(\mathbf{T}^{3}\right)^{2}\right)$, operators $U_{\mathbf{s}}$ and $H$ into direct integrals:

$$
L_{2}\left(\left(\mathbf{T}^{3}\right)^{2}\right)=\int_{\mathbf{T}^{3}} \oplus L_{2}\left(F_{\mathbf{k}}\right) d \mathbf{k}, \quad U_{\mathbf{s}}=\int_{\mathbf{T}^{3}} \oplus U_{\mathbf{s}}(\mathbf{k}) d \mathbf{k}, \quad H=\int_{\mathbf{T}^{3}} \oplus \tilde{H}(\mathbf{k}) d \mathbf{k} .
$$

Here

$$
F_{\mathbf{k}}=\left\{\left(\mathbf{k}_{\mathbf{1}}, \mathbf{k}_{\mathbf{2}}\right) \in\left(\mathbf{T}^{3}\right)^{2}: \quad \mathbf{k}_{\mathbf{1}}+\mathbf{k}_{\mathbf{2}}=\mathbf{k}\right\}
$$

$U_{\mathbf{s}}(\mathbf{k}), \mathbf{k} \in \mathbf{T}^{3}$ is the multiplication operator by the function $\exp (-i(\mathbf{s}, \mathbf{k}))$ in the space $L_{2}\left(F_{\mathbf{k}}\right)$, and fiber operators $\tilde{H}(\mathbf{k}), \mathbf{k} \in \mathbf{T}^{3}$ in $L_{2}\left(F_{\mathbf{k}}\right)$ are defined according to the formula

$$
(\tilde{H}(\mathbf{k}) f)(\mathbf{q}, \mathbf{k}-\mathbf{q})=(\mathcal{E}(\mathbf{q})+\mathcal{E}(\mathbf{k}-\mathbf{q})) f(\mathbf{q}, \mathbf{k}-\mathbf{q})-(2 \pi)^{-\frac{3}{2}} \int_{\mathbf{T}^{3}} v(\mathbf{q}-\mathbf{s}) f(\mathbf{s}, \mathbf{k}-\mathbf{s}) d \mathbf{s}
$$

and it is unitarily equivalent to the operator $H(\mathbf{k})=H_{0}(\mathbf{k})-V$, the so-called the Schrödinger operator. Unitarity is carried out using the unitary transformation:

$$
u_{\mathbf{k}}: L_{2}\left(F_{\mathbf{k}}\right) \rightarrow L_{2}\left(\mathbf{T}^{3}\right), \quad\left(u_{\mathbf{k}} g\right)(\mathbf{q})=g\left(\frac{\mathbf{k}}{2}-\mathbf{q}, \frac{\mathbf{k}}{2}+\mathbf{q}\right) .
$$

$H_{0}(\mathbf{k})$ is the multiplication operator by the function:

$$
\mathcal{E}_{\mathbf{k}}(\mathbf{q})=\mathcal{E}\left(\frac{\mathbf{k}}{2}+\mathbf{q}\right)+\mathcal{E}\left(\frac{\mathbf{k}}{2}-\mathbf{q}\right)
$$

where:

$$
\mathcal{E}(\mathbf{q})=\sum_{j=1}^{3}\left(1-\cos q^{(j)}\right)
$$

and $V$ is the integral operator in $L_{2}\left(\mathbf{T}^{3}\right)$, generated by the kernel $(2 \pi)^{-3 / 2} v(\mathbf{q}-\mathbf{s})$. The kernel $v$ of the integral operator $V$ is the Fourier transform of the potential $\hat{v}$. The potential $\hat{v}$ satisfies the conditions (1.1), therefore, the function $v$ is continuous on $\mathbf{T}^{3}$.

We denote by $N(\mathbf{k})$ the number of eigenvalues of the operator $H(\mathbf{k})$, lying to the left $\mathcal{E}_{\min }(\mathbf{k})=\min _{\mathbf{q} \in \mathbf{T}^{3}} \mathcal{E}_{\mathbf{k}}(\mathbf{q})$.

Theorem 2.1. $N(\mathbf{k}) \equiv N\left(k^{(1)}, k^{(2)}, k^{(3)}\right)$ is nondecreasing function in each $k^{(i)} \in[0, \pi]$ with other coordinates of $\mathbf{k} \in \mathbf{T}^{3}$ being fixed.

\section{Assumption 2.2. Let:}

$$
\hat{v}\left(2 s, n^{(2)}, n^{(3)}\right)=0, \forall s \in \mathbf{Z}
$$

or:

$$
\hat{v}\left(2 s+1, n^{(2)}, n^{(3)}\right)=0, \forall s \in \mathbf{Z}
$$

Theorem 2.3. Let assumption 2.2 be fulfilled. Then, each eigenvalue $z_{n}(\mathbf{k}) \equiv z_{n}\left(k^{(1)}, k^{(2)}, k^{(3)}\right)-$ of the operator $H(\mathbf{k})$ increases in $k^{(1)} \in[0, \pi]$.

Remark 2.4. Let:

$$
\hat{v}\left(n^{(1)}, 2 s, n^{(3)}\right)=0, \forall s \in \mathbf{Z}
$$

or:

(respectively

$$
\hat{v}\left(n^{(1)}, 2 s+1, n^{(3)}\right)=0, \forall s \in \mathbf{Z}
$$

$$
\hat{v}\left(n^{(1)}, n^{(2)}, 2 s\right)=0, \forall s \in \mathbf{Z}
$$

or:

$$
\left.\hat{v}\left(n^{(1)}, n^{(2)}, 2 s+1\right)=0, \forall s \in \mathbf{Z}\right) .
$$

Then, each eigenvalue $z_{n}(\mathbf{k})$ of the operator $H(\mathbf{k})$ increases in $k^{(2)} \in[0, \pi]$ (respectively in $\left.k^{(3)} \in[0, \pi]\right)$. 


\section{Eigenvalues of the two-particle operator}

Let us investigate the essential and discrete spectra of families of two-particle discrete Schrödinger operator $H(\mathbf{k}), \mathbf{k} \in \mathbf{T}^{3}$. Here, we will study the number $N(\mathbf{k})$ of eigenvalues of the operator $H(\mathbf{k})$, lying below the essential spectrum and dependence of the eigenvalues $z_{n}(\mathbf{k})$ on the total quasi-momentum $\mathbf{k} \in \mathbf{T}^{3}$.

We introduce the following notation: for a self-adjoint operator $B$ acting in a Hilbert space $\mathcal{H}$ and not having any essential spectrum on the right from the point $\mu$, denote by $\mathcal{H}_{B}(\mu) \subset \mathcal{H}, \mu \in \mathbb{R}$ subspaces such that nonzero elements $f \in \mathcal{H}_{B}(\mu)$ satisfy the inequality $(B f, f)>\mu(f, f)$ and put:

$$
n(\mu, B)=\sup _{\mathcal{H}_{B}(\mu)} \operatorname{dim} \mathcal{H}_{B}(\mu)
$$

If some point of the essential spectrum $B$ is greater $\mu$, then $n(\mu, B)$ is equal to infinity, and if $n(\mu, B)$ finite, then it is equal to the number of eigenvalues of the operator $B$, which is greater than $\mu$ (see., for example, Glazman's lemma [19])

The number $n(\mu, B)$ is the same as the number of eigenvalues of the operator $B$ lying to the right of $\mu$. For any $\mathbf{k} \in(-\pi, \pi)^{3}$ and $z \leq \mathcal{E}_{\min }(\mathbf{k})$ we define the integral operators $G(\mathbf{k}, z)$ and $Q(\mathbf{k}, z)$, acting in the space $L_{2}\left(\mathbf{T}^{3}\right)$ with the kernels:

$$
G(\mathbf{k}, z ; \mathbf{p}, \mathbf{q})=\frac{1}{(2 \pi)^{3}} \int_{\mathbf{T}^{3}} v^{\frac{1}{2}}(\mathbf{p}-\mathbf{t})\left(\mathcal{E}_{\mathbf{k}}(\mathbf{t})-z\right)^{-1} v^{\frac{1}{2}}(\mathbf{t}-\mathbf{q}) d \mathbf{t}
$$

and:

where:

$$
Q(\mathbf{k}, z ; \mathbf{p}, \mathbf{q})=(2 \pi)^{-\frac{3}{2}} v^{\frac{1}{2}}(\mathbf{p}-\mathbf{q})\left(\left(\mathcal{E}_{\mathbf{k}}(\mathbf{q})-z\right)^{\frac{1}{2}}\right)^{-1},
$$

$$
v^{\frac{1}{2}}(\mathbf{p})=\left(F \hat{v}^{\frac{1}{2}}\right)(\mathbf{p})=\frac{1}{(2 \pi)^{3 / 2}} \sum_{\mathbf{n} \in \mathbf{Z}^{3}} \sqrt{\hat{v}(\mathbf{n})} \exp (i(\mathbf{n}, \mathbf{p})) .
$$

Note that for any $z<\mathcal{E}_{\min }(\mathbf{k})$ the equalities

$$
G(\mathbf{k}, z)=V^{\frac{1}{2}} r_{0}(\mathbf{k}, z) V^{\frac{1}{2}}, \quad Q(\mathbf{k}, z)=V^{\frac{1}{2}} r_{0}^{\frac{1}{2}}(\mathbf{k}, z),
$$

hold, where $r_{0}(\mathbf{k}, z)$ is the resolvent of the unperturbed operator $H_{0}(\mathbf{k})$, and $V^{\frac{1}{2}}$ is the positive square root of the positive operator $V$. In the limiting case $z=\mathcal{E}_{\min }(\mathbf{k})$, we have the following assertion.

Lemma 3.1. For any $\mathbf{k} \in(-\pi, \pi)^{3}$ the operator $Q\left(\mathbf{k}, \mathcal{E}_{\min }(\mathbf{k})\right)$ belongs the Hilbert-Schmidt class $\Sigma_{2}$.

Proof. By virtue of conditions (1.1) for the potensial $\hat{v}(\cdot)$ the function $v^{\frac{1}{2}}(\cdot)$ belongs to $L_{2}\left(\mathbf{T}^{3}\right)$. The function $\mathcal{E}_{\mathbf{k}}(\mathbf{p})-$ $\mathcal{E}_{\min }(\mathbf{k})$ can be represented as:

$$
\mathcal{E}_{\mathbf{k}}(\mathbf{p})-\mathcal{E}_{\min }(\mathbf{k})=2 \sum_{i=1}^{3} \cos \frac{k^{(i)}}{2}\left(1-\cos p^{(i)}\right), \quad \mathbf{k} \in(-\pi, \pi)^{3},
$$

and it has only nondegenerate minimum at the point $\mathbf{p}=\mathbf{0}$, therefore:

$$
\int_{\mathbf{T}^{3}} \int_{\mathbf{T}^{3}}\left|Q\left(\mathbf{k}, \mathcal{E}_{\min }(\mathbf{k}) ; \mathbf{p}, \mathbf{q}\right)\right|^{2} d \mathbf{p} d \mathbf{q}=\frac{1}{(2 \pi)^{3}} \int_{\mathbf{T}^{3}}\left|v^{\frac{1}{2}}(\mathbf{p})\right|^{2} d \mathbf{p} \int_{\mathbf{T}^{3}} \frac{d \mathbf{q}}{\mathcal{E}_{\mathbf{k}}(\mathbf{q})-\mathcal{E}_{\min }(\mathbf{k})}<\infty .
$$

It means that $Q\left(\mathbf{k}, \mathcal{E}_{\min }(\mathbf{k})\right)$ belongs to the Hilbert-Schmidt class $\Sigma_{2}$.

From the representation $G(\mathbf{k}, z)=Q(\mathbf{k}, z)(Q(\mathbf{k}, z))^{*}$ it follows positivity and the operator $G(\mathbf{k}, z)$ belongs to the class $\Sigma_{1}$ with all $\mathbf{k} \in(-\pi, \pi)^{3}$ and $z \leq \mathcal{E}_{\min }(\mathbf{k})$.

Lemma 3.2. The number $z<\mathcal{E}_{\min }(\mathbf{k})$ is an eigenvalue of the operator $H(\mathbf{k})$ if and only if $\lambda=1$ is an eigenvalue of the operator $G(\mathbf{k}, z)$.

Proof of Theorem 2.1. Using the view (3.1) we get that the function:

$$
\left(G\left(\mathbf{k}, \mathcal{E}_{\min }(\mathbf{k})\right) \psi, \psi\right)=\int_{\mathbf{T}^{3}} \frac{\left|\left(V^{1 / 2} \psi\right)(\mathbf{p})\right|^{2} d \mathbf{p}}{\mathcal{E}_{\mathbf{k}}(\mathbf{p})-\mathcal{E}_{\min }(\mathbf{k})}=\int_{\mathbf{T}^{3}} \frac{\left|\left(V^{1 / 2} \psi\right)(\mathbf{p})\right|^{2} d \mathbf{p}}{2 \sum_{i=1}^{3} \cos \frac{k^{(i)}}{2}\left(1-\cos p^{(i)}\right)}
$$

is non-decreasing in each $k^{(i)} \in[0, \pi]$ with fixed other coordinates. This means that the function $N(\mathbf{k})$ also has this property.

Let us denote by $\lambda_{1}(\mathbf{k}, z) \geq \lambda_{2}(\mathbf{k}, z) \geq \cdots \geq \lambda_{n}(\mathbf{k}, z) \geq \cdots$ eigenvalues of the compact positive operator $G(\mathbf{k}, z)$. Each eigenvalue $\lambda_{n}(\mathbf{k}, z)$ is the even function by $k^{(i)} \in[-\pi, \pi]$. Now we will prove the monotonicity of each eigenvalue $\lambda_{n}(\mathbf{k}, z)$ by $z \in\left(-\infty, \mathcal{E}_{\min }(\mathbf{k})\right)$ and $k^{(i)} \in[0, \pi]$. 
The following lemma is a Birman-Schwinger principle for the operator $H(\mathbf{k})$.

Lemma 3.3. For any $\mathbf{k} \in(-\pi, \pi)^{3}$ and $z \leq \mathcal{E}_{\min }(\mathbf{k})$ the equality:

holds.

$$
n(-z,-H(\mathbf{k}))=n(1, G(\mathbf{k}, z)),
$$

Proof. A proof of a similar lemma is given in the paper [15].

Lemma 3.4. For any $\mathbf{k} \in(-\pi, \pi)^{3}$ each positive eigenvalue $\lambda_{n}(\mathbf{k}, z)$ of the operator $G(\mathbf{k}, z)$ increases by $z \in$ $\left(-\infty, \mathcal{E}_{\min }(\mathbf{k})\right)$.

Proof. For any $\psi \in L_{2}\left(\mathbf{T}^{3}\right)$ and $z_{1}<z_{2} \in\left(-\infty, \mathcal{E}_{\min }(\mathbf{k})\right)$ the inequality holds

$$
\int_{\mathbf{T}^{3}} \frac{\left|\left(V^{1 / 2} \psi\right)(\mathbf{p})\right|^{2} d \mathbf{p}}{\mathcal{E}_{\mathbf{k}}(\mathbf{p})-z_{1}} \leq \int_{\mathbf{T}^{3}} \frac{\left|\left(V^{1 / 2} \psi\right)(\mathbf{p})\right|^{2} d \mathbf{p}}{\mathcal{E}_{\mathbf{k}}(\mathbf{p})-z_{2}}
$$

Hence $\left(G\left(\mathbf{k}, z_{1}\right) \psi, \psi\right) \leq\left(G\left(\mathbf{k}, z_{2}\right) \psi, \psi\right)$, so $\lambda_{n}\left(\mathbf{k}, z_{1}\right) \leq \lambda_{n}\left(\mathbf{k}, z_{2}\right)$. Now, we show the strict inequality:

$$
\lambda_{n}\left(\mathbf{k}, z_{1}\right)<\lambda_{n}\left(\mathbf{k}, z_{2}\right) .
$$

Let $\mathcal{H}_{\left[\lambda_{n}, \infty\right)}\left(G\left(\mathbf{k}, z_{1}\right)\right)$ be subspace generated by the eigenfunctions of the operator $G\left(\mathbf{k}, z_{1}\right)$, corresponding eigenvalues $\lambda_{1}\left(\mathbf{k}, z_{1}\right) \geq \lambda_{2}\left(\mathbf{k}, z_{1}\right) \geq \ldots \geq \lambda_{n}\left(\mathbf{k}, z_{1}\right)>0$. For any non-zero $\psi \in \mathcal{H}_{\left[\lambda_{n}, \infty\right)}\left(G\left(\mathbf{k}, z_{1}\right)\right)$ we obtain:

$$
\left(G\left(\mathbf{k}, z_{2}\right) \psi, \psi\right)=\int_{\mathbf{T}^{3}} \frac{\left|\left(V^{1 / 2} \psi\right)(\mathbf{p})\right|^{2} d \mathbf{p}}{\mathcal{E}_{\mathbf{k}}(\mathbf{p})-z_{2}}>\int_{\mathbf{T}^{3}} \frac{\left|\left(V^{1 / 2} \psi\right)(\mathbf{p})\right|^{2} d \mathbf{p}}{\mathcal{E}_{\mathbf{k}}(\mathbf{p})-z_{1}}=\left(G\left(\mathbf{k}, z_{1}\right) \psi, \psi\right) \geq \lambda_{n}\left(\mathbf{k}, z_{1}\right)(\psi, \psi) .
$$

Hence, strict inequality (3.3) holds.

Lemma 3.5. Let assumption 2.2 be fulfilled. Then, for any $z \in\left(-\infty, \mathcal{E}_{\min }(\mathbf{k})\right)$, each positive eigenvalue $\lambda_{n}(\mathbf{k}, z)$ of the operator $G(\mathbf{k}, z)$ decreases in $k^{(1)} \in[0, \pi]$.

Proof. Let the condition (2.1) be satisfied. Then for the function $v^{\frac{1}{2}}(\mathbf{p})$, the following equality

$$
v^{\frac{1}{2}}\left(p^{(1)}+\pi, p^{(2)}, p^{(3)}\right)=-v^{\frac{1}{2}}\left(p^{(1)}, p^{(2)}, p^{(3)}\right)
$$

holds. Similarly, if satisfing the condition (2.2), then

$$
v^{\frac{1}{2}}\left(p^{(1)}+\pi, p^{(2)}, p^{(3)}\right)=v^{\frac{1}{2}}\left(p^{(1)}, p^{(2)}, p^{(3)}\right) .
$$

Therefore, in both cases $\left|\left(V^{\frac{1}{2}} \psi\right)(\mathbf{p})\right|=|\varphi(\mathbf{p})|$ is a $\pi$ - periodic function by argument $p^{(1)}$. For any $\psi \in L_{2}\left(\mathbf{T}^{3}\right)$ we have

$$
(G(\mathbf{k}, z) \psi, \psi)=\int_{\mathbf{T}^{3}} \frac{|\varphi(\mathbf{p})|^{2} d \mathbf{p}}{\mathcal{E}_{\mathbf{k}}(\mathbf{p})-z}=\int_{\mathbf{T}^{2}}\left\{\int_{-\pi}^{\pi} \frac{|\varphi(\mathbf{p})|^{2} d p^{(1)}}{B\left({ }^{\prime} \mathbf{k},{ }^{\prime} \mathbf{p} ; z\right)-2 \cos \frac{k^{(1)}}{2} \cos p^{(1)}}\right\} d^{\prime} \mathbf{p} .
$$

Here, ${ }^{\prime} \mathbf{k}=\left(k^{(2)}, k^{(3)}\right),{ }^{\prime} \mathbf{p}=\left(p^{(2)}, p^{(3)}\right) \in \mathbf{T}^{2}$,

$$
B\left({ }^{\prime} \mathbf{k},{ }^{\prime} \mathbf{p} ; z\right)=6-2 \cos \frac{k^{(2)}}{2} \cos p^{(2)}-2 \cos \frac{k^{(3)}}{2} \cos p^{(3)}-z>0, \quad z<\mathcal{E}_{\min }(\mathbf{k}) .
$$

The inner integral of the right-hand side of the equality (3.4) is represented as the sum of two integrals over the intervals $[-\pi, 0]$ and $[0, \pi]$. In the first integral, making the replacement variable $p^{(1)}=\pi+q^{(1)}$ and using the identity $\cos (\pi+x)=-\cos x$ and property $\left|\varphi\left(p^{(1)}+\pi,{ }^{\prime} \mathbf{p}\right)\right|=|\varphi(\mathbf{p})|$ we have:

$$
(G(\mathbf{k}, z) \psi, \psi)=2 \int_{\mathbf{T}^{2}} B\left({ }^{\prime} \mathbf{k},{ }^{\prime} \mathbf{p} ; z\right)\left\{\int_{0}^{\pi} \frac{|\varphi(\mathbf{p})|^{2} d p^{(1)}}{B^{2}\left({ }^{\prime} \mathbf{k},{ }^{\prime} \mathbf{p} ; z\right)-4 \cos ^{2} \frac{k^{(1)}}{2} \cos ^{2} p^{(1)}}\right\} d^{\prime} \mathbf{p} .
$$

Since $B\left({ }^{\prime} \mathbf{k},{ }^{\prime} \mathbf{p} ; z\right)>0$ with all ' $\mathbf{p} \in \mathbf{T}^{2}, z<\mathcal{E}_{\min }(\mathbf{k})$, the inner integral in (3.5) strictly decreases with increasing $k^{(1)} \in[0, \pi]$. The monotonicity of the integral implies that:

$$
(G(\mathbf{k}, z) \psi, \psi)>\left(G\left(\mathbf{k}^{\prime}, z\right) \psi, \psi\right)
$$

if $\varphi=V^{1 / 2} \psi$ is a nonzero element in $L_{2}\left(\mathbf{T}^{3}\right)$ and $\mathbf{k}=\left(k^{(1)}, k^{(2)}, k^{(3)}\right), \mathbf{k}^{\prime}=\left(k_{1}^{(1)}, k^{(2)}, k^{(3)}\right)$ at $0 \leq k^{(1)}<$ $k_{1}^{(1)} \leq \pi$. Note that from the inclusion $\psi \in \mathcal{H}_{\left[\lambda_{n}, \infty\right)}\left(G\left(\mathbf{k}^{\prime}, z\right)\right) \backslash\{0\}$ it follows that $V^{1 / 2} \psi \neq 0$. Therefore, from 
the inequality (3.6), the assertion $(G(\mathbf{k}, z) \psi, \psi) \geq \lambda_{n}\left(\mathbf{k}^{\prime}, z\right)(\psi, \psi)$ holds for all $\psi \in \mathcal{H}_{\left[\lambda_{n}, \infty\right)}\left(G\left(\mathbf{k}^{\prime}, z\right)\right) \backslash\{0\}$. This proves that $\lambda_{n}(\mathbf{k}, z)>\lambda_{n}\left(\mathbf{k}^{\prime}, z\right)$ by $0 \leq k^{(1)}<k_{1}^{(1)} \leq \pi$.

Proof of Theorem 2.3. Let $\mathbf{k}=\left(k^{(1)}, k^{(2)}, k^{(3)}\right)$ and $\mathbf{k}_{\mathbf{1}}=\left(k_{1}^{(1)}, k^{(2)}, k^{(3)}\right)$ be two arbitrary points such that $0 \leq k^{(1)}<k_{1}^{(1)} \leq \pi$. Let the operator $H(\mathbf{k})$ has $N=N(\mathbf{k})$ eigenvalues $z_{1}(\mathbf{k}) \leq z_{2}(\mathbf{k}) \leq \cdots \leq z_{N}(\mathbf{k})$, lying below $\mathcal{E}_{\min }(\mathbf{k})$. Existence is not less than $N(\mathbf{k})$ eigenvalues of the operator $H\left(\mathbf{k}_{\mathbf{1}}\right)$ follows from Theorem 2.1. From here, it follows that the operator $G(\mathbf{k}, z)$ has $N(\mathbf{k})$ eigenvalues:

$$
\lambda_{1}(\mathbf{k}, z) \geq \lambda_{2}(\mathbf{k}, z) \geq \cdots \geq \lambda_{N}(\mathbf{k}, z)>1
$$

by $z \in\left(z_{N}(\mathbf{k}), \mathcal{E}_{\min }(\mathbf{k})\right]$. The continuity of $G(\mathbf{k}, z)$ with respect to the totality of variables $\mathbf{k} \in \mathbf{T}^{3}$ and $z<\mathcal{E}_{\min }(\mathbf{k})$ implies the continuity of $\lambda_{n}(\mathbf{k}, z), 1 \leq n \leq N$, with respect such arguments $\mathbf{k}$ and $z$. It is easy to show that

$$
\lim _{z \rightarrow-\infty}\|G(\mathbf{k}, z)\|=0 .
$$

From the inequality $\lambda_{n}(\mathbf{k}, z) \leq\|G(\mathbf{k}, z)\|$ it follows that for any $n \in\{1,2, \ldots, N\}$ the equation $\lambda_{n}(\mathbf{k}, z)=1$ has a unique solution $z=z_{n}(\mathbf{k}) \in\left(-\infty, \mathcal{E}_{\min }(\mathbf{k})\right)$. Uniqueness follows from the monotonicity of $\lambda_{n}(\mathbf{k}, \cdot)$ in $\left(-\infty, \mathcal{E}_{\min }(\mathbf{k})\right)$. By virtue of Lemma 3.2, the number $z_{n}(\mathbf{k})$ is the eigenvalue of the operator $H(\mathbf{k})$. Using the definition of $z_{n}(\mathbf{k})$, the inequality $\lambda_{n}(\mathbf{k}, z) \geq \lambda_{n+1}(\mathbf{k}, z)$ and monotonicity of the function $\lambda_{n}(\mathbf{k}, \cdot)$ we obtain that $z_{n}(\mathbf{k}) \leq z_{n+1}(\mathbf{k}), n=\overline{1, N-1}$. Now, let's show the monotonicity of $z_{n}(\mathbf{k})$ in each $k^{(1)} \in[0, \pi]$. By virtue of Lemma 3.5 an eigenvalue $\lambda_{n}(\mathbf{k}, z)$ is the decreasing function with respect to $k^{(1)} \in[0, \pi]$, and hence:

$$
1=\lambda_{n}\left(\mathbf{k}, z_{n}(\mathbf{k})\right)>\lambda_{n}\left(\mathbf{k}_{\mathbf{1}}, z_{n}(\mathbf{k})\right) .
$$

On the other side:

$$
1=\lambda_{n}\left(\mathbf{k}_{\mathbf{1}}, z_{n}\left(\mathbf{k}_{\mathbf{1}}\right)\right)>\lambda_{n}\left(\mathbf{k}_{\mathbf{1}}, z_{n}(\mathbf{k})\right) .
$$

Since $\lambda_{n}(\mathbf{k}, \cdot)$ is an increasing function in $\left(-\infty, \mathcal{E}_{\min }(\mathbf{k})\right)$, we get $z_{n}\left(\mathbf{k}_{\mathbf{1}}\right)>z_{n}(\mathbf{k})$.

Notice that the assumption (2.1) is essential. The following example shows that if the assumption (2.1) is not satisfied, then there is a potential $\hat{v}$ and the segment $[\pi-\delta, \pi]$, such that the eigenvalue $E_{0}(\mathbf{k})$ of the operator $H(\mathbf{k})$ strictly decreases in $k^{(1)} \in[\pi-\delta, \pi]$.

Example 3.6. Let $\hat{v}(\mathbf{0})=2 \hat{v}\left(\mathbf{e}_{1}\right)=2 \hat{v}\left(-\mathbf{e}_{1}\right)=2, \hat{v}(\mathbf{n})=0$ at $\mathbf{n} \neq \mathbf{0}, \mathbf{n} \neq \pm \mathbf{e}_{1}$. Then the operator $H(\pi, \pi, \pi)$ has simple eigenvalue $E_{0}=4$. Using perturbation theory, we obtain that the operator $H(\pi-\beta, \pi, \pi)$ has a unique simple eigenvalue $E_{0}(\pi-\beta, \pi, \pi)$ in the neighborhood of $E_{0}$ for small $\beta$, and for $E_{0}(\pi-\beta, \pi, \pi)$ the following asymptotic formula holds [20]:

$$
E_{0}(\pi-\beta, \pi, \pi)=E_{0}-\frac{\hat{v}(\mathbf{0})-3 \hat{v}\left(\mathbf{e}_{1}\right)}{\hat{v}(\mathbf{0})-\hat{v}\left(\mathbf{e}_{1}\right)} \frac{1}{4} \beta^{2}+O\left(\beta^{4}\right) \quad \text { at } \quad \beta \rightarrow 0 .
$$

This implies the existence of the segment $[\pi-\delta, \pi]$, where $E_{0}\left(k^{(1)}, \pi, \pi\right)$ strictly decreases.

\section{Conclusion}

We study the two-particle Schrödinger operator $H(\mathbf{k}),\left(\mathbf{k} \in \mathbf{T}^{3} \equiv(-\pi, \pi]^{3}\right.$ is the total quasimomentum of a system of two particles) corresponding to the Hamiltonian of the two-particle system on the three-dimensional lattice $\mathbf{Z}^{3}$. We prove that the number $N(\mathbf{k}) \equiv N\left(k^{(1)}, k^{(2)}, k^{(3)}\right)$ of eigenvalues below the essential spectrum of the operator $H(\mathbf{k})$ is nondecreasing function in each $k^{(i)} \in[0, \pi], i=1,2,3$. We show the monotonicity property of each eigenvalue $z_{n}(\mathbf{k}) \equiv z_{n}\left(k^{(1)}, k^{(2)}, k^{(3)}\right)$ of the operator $H(\mathbf{k})$ in $k^{(i)} \in[0, \pi]$ with other coordinates $\mathbf{k}$ being fixed.

In [21], for the case $d=1$ and $\operatorname{card}\{n \in \mathbf{Z}: \hat{v}(n)>0\}=\infty$, the limit result:

$$
\lim _{k \rightarrow \pi-} N(k)=+\infty
$$

for the number $N(k)$ of the eigenvalues of the operator $H(k)$ was proved. We remark that in our case if $\operatorname{card}\{\mathbf{n} \in$ $\left.\mathbf{Z}^{3}: \hat{v}(\mathbf{n})>0\right\}=\infty$, then one can prove the above limit result.

In the following, we give some generalizations of the statement of Theorem 2.3. If the potential $\hat{v}$ satisfies one of the conditions:

$$
\begin{gathered}
\hat{v}\left(2 s^{(1)}+1,2 s^{(2)}+1,2 s^{(3)}+1\right)=0, \quad \forall s=\left(s^{(1)}, s^{(2)}, s^{(3)}\right) \in \mathbf{Z}^{\mathbf{3}}, \\
\hat{v}\left(2 s^{(1)}, 2 s^{(2)}, 2 s^{(3)}\right)=0, \quad \forall s=\left(s^{(1)}, s^{(2)}, s^{(3)}\right) \in \mathbf{Z}^{\mathbf{3}},
\end{gathered}
$$

then the eigenvalue $z_{n}\left(k^{(1)}, k^{(2)}, k^{(3)}\right)$ of the operator $H(\mathbf{k})$ increases with respect to each argument $k^{(1)}, k^{(2)}$ and $k^{(3)}$ in $[0, \pi]$. 
One can show that the statement of Theorem 2.3 is preserved, if the dimension $d$ of the lattice $\mathbf{Z}^{d}$ is greater than three. It is clear that for $d=1,2$, is impossible to define the Birman-Schwinger operator $G(\mathbf{k}, z)$ in the whole space at the point $z=\mathcal{E}_{\min }(\mathbf{k})$. Let us denote by:

$$
L_{2}^{e}\left(\mathbf{T}^{d}\right)=\left\{f \in L_{2}\left(\mathbf{T}^{d}\right): f(-\mathbf{p})=f(\mathbf{p})\right\} \quad \text { and } \quad L_{2}^{o}\left(\mathbf{T}^{d}\right)=\left\{f \in L_{2}\left(\mathbf{T}^{d}\right): f(-\mathbf{p})=-f(\mathbf{p})\right\} .
$$

For the even potential $\hat{v}$, the subspaces $L_{2}^{e}\left(\mathbf{T}^{d}\right)$ and $L_{2}^{o}\left(\mathbf{T}^{d}\right)$ are invariant under the operator $H(\mathbf{k})$. The operator $G^{o}(\mathbf{k}, z)$, corresponding to the operator $H^{o}(\mathbf{k})=\left.H(\mathbf{k})\right|_{L_{2}^{o}\left(\mathbf{T}^{d}\right)}$, can be defined as a compact operator on the boundary $z=\mathcal{E}_{\min }(\mathbf{k})$ of the essential spectrum. In this case, one can prove a similar result concerning to the monotonicity of the eigenvalues of the operator $H^{o}(\mathbf{k})$ with respect to $k^{(i)} \in[0, \pi]$.

\section{Acknowledgement}

The authors expresses gratitude to the referee for valuable remarks.

\section{References}

[1] Bloch I., Dalibard J., and Nascimbene S. Quantum simulations with ultracold quantum gases, Nature Physics, 2012, 8, P. $267-276$.

[2] Jaksch D., Zoller P. The cold atom Hubbard toolbox, Annals of Physics, 2005, 315, P. 52-79.

[3] Lewenstein M., Sanpera A., Ahufinger V. Ultracold Atoms in Optical Lattices: Simulating Quantum Many-body Systems, 2012, Oxford University Press.

[4] Gullans M., Tiecke T.G., Chang D.E., Feist J., Thompson J.D., Cirac J.I., Zoller P., Lukin M.D. Nanoplasmonic Lattices for Ultracold Atoms, Phys. Rev. Lett., 2012, 109, P. 235309.

[5] Hecht E. Optics. Addison-Wesley, Reading, MA, 1998.

[6] Murphy B., Hau L.V. Electro-optical nanotraps for neutral atoms, Phys. Rev. Lett., 2009, 102, P. 033003.

[7] N.P. de Leon, Lukin M D., and Park H. Quantum plasmonic circuits, IEEE J. Sel. Top. Quantum Electron., 2012, 18, P. $1781-1791$.

[8] Mattis D.C. The few-body problem on a lattice. Rev. Modern Phys., 1986, 58, P. 361-379.

[9] Mogilner A.I. Hamiltonians in solid-state physics as multiparticle discrete Schrödinger operators: Problems and results, in: Many Particle Hamiltonians: Spectra and Scattering (Adv. Soviet Math., Vol. 5, R. A. Minlos, ed.), Amer. Math. Soc., Providence, R.I., 1991, P. $139-194$.

[10] Graf G.M., Schenker D. 2-magnon scattering in the Heisenberg model, Ann. Inst. H. Poincare' Phys. The'or, 1997, 67(1), P. 91-107.

[11] Muminov M.I., Ghoshal S.K. Spectral features of two-particle Schrödinger operator on d-dimensiional lattice. Complex Analysis and Operator Theory, 2020, 14(1).

[12] Lakaev S.N., Khalkhuzhaev A.M. The number of eigenvalues of the two-particle discrete Schrödinger operator. Theor. Math. Phys., 2009, 158(2), P. 221-232.

[13] Abdullaev J.I., Lakaev S.N. Asymptotics of the discrete spectrum of the three-particle Schrödinger difference operator on lattice, Theor. Math. Phys., 2003, 136(2), P. 1096-1109.

[14] Albeverio S., Lakaev S.N., Makarov K.A., Muminov Z.I. The threshold effects for the two-particle Hamiltonians on lattices, Commun. Math. Phys., 2006, 262(1), P. 91-115.

[15] Lakaev S.N., Khalkhuzhaev A.M. Spectrum of the two-particle Schrödinger Operator on a lattice, Theor. Math. Phys., 2008, 155(2), P. 753764.

[16] Lakaev S., Kholmatov Sh., Khamidov Sh. Bose-Hubbard models with on-site and nearest-neighbor interactions: Exactly solvable case. $J$. Phys. A: Math. Theor., 2021, 54, P. 245201(22).

[17] Muminov M., Lokman C. Finiteness of discrete spectrum of the two-particle Schrödinger operator on diamond lattices, Nanosystems: physics, chemistry, mathematics, 2017, 8(3), P. 310-316.

[18] M. Reed and B. Simon, Methods of modern mathematical physics. IV: Analysis of operators. Academic Press, N.Y., 1979.

[19] Pankov A.A. Lecture Notes on Schrödinger equations. Nova Science, New York, 2007.

[20] Abdullaev Zh.I., Kuliev K.D. Bound States of a two-boson system on a two-dimensional lattice, Theor. Math. Phys., 2016, 186(2), P. $231-250$.

[21] Abdullaev Zh.I. Bound states of a system of two fermions on a one-dimensional lattice. Theor. and Math. Phys., 2006, 147(1), P. 486-495. 